\title{
M DWARF METALLICITIES AND GIANT PLANET OCCURRENCE: IRONING OUT UNCERTAINTIES AND SYSTEMATICS
}

\author{
ErIC GAIDOs $^{1,3}$ AND ANDrew W. MANN ${ }^{2,3,4}$ \\ ${ }^{1}$ Department of Geology \& Geophysics, University of Hawaii at Mānoa, Honolulu, HI 96822, USA; gaidos@ hawaii.edu \\ ${ }^{2}$ Department of Astronomy, University of Texas at Austin, Austin, TX 78712, USA \\ Received 2014 May 14; accepted 2014 June 18; published 2014 July 25
}

\begin{abstract}
Comparisons between the planet populations around solar-type stars and those orbiting $\mathrm{M}$ dwarfs shed light on the possible dependence of planet formation and evolution on stellar mass. However, such analyses must control for other factors, i.e., metallicity, a stellar parameter that strongly influences the occurrence of gas giant planets. We obtained infrared spectra of $121 \mathrm{M}$ dwarfs stars monitored by the California Planet Search and determined metallicities with an accuracy of 0.08 dex. The mean and standard deviation of the sample are -0.05 and 0.20 dex, respectively. We parameterized the metallicity dependence of the occurrence of giant planets on orbits with a period less than two years around solar-type stars and applied this to our $\mathrm{M}$ dwarf sample to estimate the expected number of giant planets. The number of detected planets (3) is lower than the predicted number (6.4), but the difference is not very significant (12\% probability of finding as many or fewer planets). The three $\mathrm{M}$ dwarf planet hosts are not especially metal rich and the most likely value of the power-law index relating planet occurrence to metallicity is 1.06 dex per dex for M dwarfs compared to 1.80 for solar-type stars; this difference, however, is comparable to uncertainties. Giant planet occurrence around both types of stars allows, but does not necessarily require, a mass dependence of $\sim 1$ dex per dex. The actual planet-mass-metallicity relation may be complex, and elucidating it will require larger surveys like those to be conducted by ground-based infrared spectrographs and the Gaia space astrometry mission.
\end{abstract}

Key words: planets and satellites: formation - planets and satellites: gaseous planets - stars: abundances - stars: fundamental parameters - stars: low-mass - techniques: spectroscopic

Online-only material: color figures, machine-readable table

\section{INTRODUCTION}

The formation of gas giant planets like Jupiter is a central problem in planetary science. In the prevailing scenario, a solid core of rock and ice is the nucleation site for the runaway accretion of a gaseous envelope (Helled et al. 2013). This model predicts that planet-forming disks with more condensable elements form cores more readily and that metal-rich stars are more likely to host giant planets. This correlation has been confirmed by Doppler radial velocity (RV) surveys of solartype stars (Gonzalez 1998; Fischer \& Valenti 2005), and all evidence points to a similar correlation for M dwarfs (Johnson \& Apps 2009; Neves et al. 2013; Mann et al. 2013c).

Stellar mass may also be an important determinant of giant planet formation. If more massive stars are born with more massive disks, which is something suggested but not unambiguously supported by observations (Andrews et al. 2013), giant planet occurrence should also increase with stellar mass. Cumming et al. (2008) analyzed Doppler RV detections in the California Planet Search (CPS) and estimated that M dwarfs have 3-10 times fewer giant planets within 2.5 AU than solartype stars. Johnson et al. (2010) re-visited the stellar mass and metallicity distributions of CPS-detected giant planets around both solar-mass and M dwarf stars and found that (1) all M dwarf giant planet hosts are metal-rich, and (2) accounting for metallicity, $\mathrm{M}$ dwarfs host about half as many giant planets as do

\footnotetext{
3 Visiting Astronomer at the Infrared Telescope Facility, which is operated by the University of Hawaii under Cooperative Agreement no. NNX-08AE38A with the National Aeronautics and Space Administration, Science Mission Directorate, Planetary Astronomy Program.

4 Harlan J. Smith Postdoctoral Fellow.
}

solar-mass stars. Conversely, they found that giant planets are found even more frequently around evolved stars that derive from more massive progenitors.

More recently, Montet et al. (2014) used adaptive optics imaging to rule out stellar companions to $\mathrm{M}$ dwarfs in cases where a long-term drift in RV was found by Doppler observations. This allowed them to better constrain the occurrence of giant planets at separations to $\sim 20 \mathrm{AU}$ from $111 \mathrm{M}$ dwarfs monitored by the CPS. Combining this with survey data for higher mass stars, they found a strong metallicity dependence ( $3.8 \pm 1.2 \mathrm{dex}$ per dex) but a much weaker and less significant dependence on mass $(0.8 \pm 1$ dex per dex). Moreover, within their $\mathrm{M}$ dwarf sample they found occurrence to decrease with mass by a factor of 2.7 relative to the prediction of the universal relation of Johnson et al. (2010), albeit at low statistical significance. Clanton \& Gaudi (2014) combined microlensing and RV surveys to probe planet occurrence at similar distances and found that $\mathrm{M}$ dwarfs have two to three times fewer giant planets than FGK dwarfs. However, part of this difference is a result of their assumption that comparisons should be scaled by the theoretical "ice line" in the protoplanetary disk. This is expected to lie at greater distances from solar-type stars than M dwarfs, and thus, such a scaling includes planets on a wider range of separations around the former.

Gaidos et al. (2013) reported an occurrence of $4.0 \% \pm 2.3 \%$ for Doppler-detected giant planets with an orbital period $P<$ 245 days orbiting late $\mathrm{K}$ dwarfs. If extrapolated to the separation range considered by Johnson et al. (2010) using a flat log-period distribution, this corresponds to an occurrence of $5.5 \% \pm 3 \%$. Gaidos et al. (2013) also performed a linear least-squares regression of giant planet occurrence versus representative 
stellar mass from published Doppler surveys. The fit predicts a quadrupling of the occurrence from $\mathbf{M}$ dwarfs to solar-mass stars, but the scatter is so large that the significance of any mass dependence is weak ( $F$-test, $p=0.12$ ).

One wrinkle in this story is the suggestion of Lloyd (2011) that the masses assigned to the evolved stars in the Johnson et al. (2010) sample $\left(M_{*}>1.5 M_{\odot}\right)$ have been overestimated and are only slightly larger than solar. The crux of the argument revolves around whether there are too many high-mass stars in Doppler surveys compared to plausible stellar population models (Lloyd 2013; Johnson et al. 2013). Schlaufman \& Winn (2013) found that the velocity dispersion of the stars in question were consistent with an older age, and thus progenitors with a lower mass comparable to the solar value. This could mean that the trend with mass observed by Johnson et al. (2010) is the result of a sensitive threshold effect, an artifact of age and orbital evolution, and/or the result of a high false positive rate among evolved stars.

Another wrinkle is a systematic that arises when stars of different types are compared: conflation of the effects of mass and metallicity. The sensitivity of giant planet occurrence to metallicity means that comparisons between stars of different masses must carefully control for this parameter: an offset of only $0.1-0.2$ dex in $[\mathrm{Fe} / \mathrm{H}]$ could equate to a factor of two in occurrence (Neves et al. 2013). The metallicity distribution of M dwarfs in Doppler surveys need not necessarily be identical to that of solar-type stars in the solar neighborhood because of differences in age and formation location or, because metallicity and luminosity are related, due to selection bias. The metallicities of M dwarfs have not been, until recently, determined to the necessary precision, partly because the strength of metallicitysensitive features is difficult to determine at visible wavelengths because of overlapping lines and the lack of a well-defined continuum (e.g., Mann et al. 2013a; Pineda et al. 2013). In addition, calibrations based on stars with independently established metallicities did not exist. This situation has been ameliorated with the proliferation of infrared spectrographs that probe a wavelength range $(1.2-2.5 \mu \mathrm{m})$ of $\mathrm{M}$ dwarf spectra where there are isolated metallicity-sensitive lines and a well-defined continuum (Rojas-Ayala et al. 2012a; Önehag et al. 2012; Terrien et al. 2012; Mann et al. 2013a).

To address the possible conflation of metallicity and mass dependence, we obtained infrared spectra of most of the M dwarfs monitored by the CPS. We also obtained visible-wavelength spectra of about half the sample to make new empirical estimates of stellar parameters. We describe the observations and data reduction in Section 2; describe the resulting distributions of metallicities and masses in Section 3; analyze and compare planet occurrence to the well-characterized solar-mass stars in the Spectroscopic Properties of Cool Stars (SPOCSs; Valenti $\&$ Fischer 2005) in Section 4; and discuss our findings, their caveats, and their implications in Section 5.

\section{OBSERVATIONS AND REDUCTION}

\subsection{SpeX Infrared Spectra}

Using the SpeX spectrograph (Rayner et al. 2003) on the NASA Infrared Telescope (IRTF), we obtained simultaneous $J H K$ spectra of 121 of the 147 stars in the Rauscher \& Marcy (2006, hereafter RM06) catalog of M dwarfs monitored by the CPS. Observations were carried out between 2011 September and 2013 December and targets were selected entirely based on visibility from Maunakea, i.e., without regard to their properties or whether they host known planets. We used SpeX in the crossdispersed (SXD) mode and the 0.3 arcsec slit, which yielded simultaneous coverage from 0.8 to $2.4 \mu \mathrm{m}$ at a resolution $\lambda / \Delta \lambda$ of about 2000. Targets were nodded between two positions along the slit (A and B) to ensure accurate sky subtraction. At least six exposures were taken of each target following the ABBA nodding pattern. Exposure times are capped at $120 \mathrm{~s}$ to mitigate errors from atmospheric variation, although most exposure times were much shorter (typically 10-20 s). This was sufficient to ensure a signal-to-noise ratio $(\mathrm{S} / \mathrm{N})$ of $>60 \mathrm{pixel}^{-1}$ in the $K$-band, and $>80$ pixel $^{-1}$ in the $H$-band for all targets. We observed an A0V-type star, used to remove telluric lines, within $1 \mathrm{hr}$ of hour angle and 0.1 airmasses of each target.

Reduction was carried out using the SpeXtool IDL package (Cushing et al. 2004), which performed bias and flat-field corrections, wavelength calibration, sky subtraction, and extraction of the one-dimensional spectrum. Multiple exposures were stacked using the xcombspec routine. Telluric corrections were derived for each target using the relevant A0V star observation and the xtellcor package (Vacca et al. 2003). Reduced spectra were put in vacuum wavelengths and shifted to their rest wavelengths using template stars from the IRTF spectral library (Cushing et al. 2005; Rayner et al. 2009).

Metallicities of the $\mathrm{M}$ dwarfs were calculated following the procedure of Mann et al. (2013a). They provide empirical relations between the metallicity of $\mathrm{M}$ dwarfs and the strength of atomic lines in visible, $\mathrm{J}$-, $\mathrm{H}$-, and $\mathrm{K}$-band spectra, calibrated using wide binaries with an $\mathrm{F}, \mathrm{G}$, or $\mathrm{K}$ dwarf primary and an M dwarf companion. Metallicities of the FGK primaries were forced to match the metallicity scale from SPOCS (Valenti \& Fischer 2005) ensuring that the M dwarf metallicities are on the same scale. We derived metallicities using the weighted mean of the $H$ and $K$ band relations, accounting for both measurement and errors in the calibration. Because the $\mathrm{S} / \mathrm{N}$ of our SpeX data is typically high $\left(>100\right.$ pixel $^{-1}$ ) errors are dominated by the Mann et al. (2013a) calibration errors, and the gain of adding in the visible and $J$-band relations (which have higher errors) is negligible. Resulting metallicities are reported in Table 1.

\subsection{SNIFS Visible Wavelength Spectra}

We obtained visible wavelength spectra of 72 of the 147 stars in the Rauscher \& Marcy (2006) sample using the SuperNova Integral Field Spectrograph (SNIFS; Aldering et al. 2002; Lantz et al. 2004) attached to the University of Hawaii $2.2 \mathrm{~m}$ telescope atop Maunakea. SNIFS utilizes separate blue (3200-5200 А) and red (5100-9700 $\AA$ ) channels separated by a dichroic at resolutions of $R \sim 800$ and $R \sim 1000$, respectively. Observations were done between 2010 July and 2012 December, primarily as part of spectroscopic followup of the Lépine \& Gaidos (2011) sample (Gaidos et al. 2014). Exposure times ranged from 30 to $270 \mathrm{~s}$, which provided peak $\mathrm{S} / \mathrm{N}$ of $>120 \mathrm{pixel}^{-1}$ in the red channel for all targets. Although the $\mathrm{S} / \mathrm{N}$ was lower in the blue channel, these data were not used for our analysis.

Basic SNIFS reduction, including bias and flat field corrections, wavelength calibration, masking cosmic rays and bad pixels, and extracting the one-dimensional spectrum from the data cube, were performed by the SNIFS reduction pipeline as described in Bacon et al. (2001) and Aldering et al. (2006). Spectra were then flux calibrated using the atmosphere model from Buton et al. (2013) combined with spectrophotometric standards observed throughout the night (Oke 1990; Hamuy et al. 1994; Bohlin et al. 2001). Spectra were then shifted to their rest frames by putting the wavelengths in vacuum, then 
Table 1

Parameters of nearby M Dwarfs from the California Planet Search

\begin{tabular}{|c|c|c|c|c|c|c|c|}
\hline \multirow[t]{2}{*}{ Name } & \multirow[t]{2}{*}{ HIP } & \multirow[t]{2}{*}[\mathrm{Fe}/\mathrm{H}]{} & $T_{\text {eff }}(\mathrm{K})$ & $R_{*}\left(R_{\odot}\right)$ & $L_{*}\left(L_{\odot}\right)$ & $M_{*}\left(M_{\odot}\right)$ & $T_{\text {eff }}$ src \\
\hline & & & \multicolumn{5}{|c|}{ (Negative Values are Upper Limits) } \\
\hline GJ 2 & 428 & $-0.01 \pm 0.08$ & $3781 \pm 73$ & $0.52 \pm 0.04$ & $0.0482 \pm 0.0091$ & $0.56 \pm 0.07$ & $K$-band \\
\hline HD 225213 & 439 & $-0.39 \pm 0.08$ & $3401 \pm 73$ & $0.32 \pm 0.06$ & $0.0131 \pm 0.0051$ & $0.31 \pm 0.07$ & $K$-band \\
\hline LHS 1053 & 1368 & $0.07 \pm 0.08$ & $4336 \pm 73$ & $0.67 \pm 0.04$ & $0.1462 \pm 0.0191$ & $0.69 \pm 0.07$ & $K$-band \\
\hline GX And & 1475 & $-0.26 \pm 0.08$ & $3693 \pm 91$ & $0.49 \pm 0.05$ & $0.0382 \pm 0.0099$ & $0.52 \pm 0.07$ & Visible \\
\hline GQ And & 0 & $-0.20 \pm 0.08$ & $3254 \pm 71$ & $0.20 \pm 0.07$ & $0.0041 \pm 0.0039$ & $0.16 \pm 0.09$ & Visible \\
\hline BPM 46239 & 1734 & $0.27 \pm 0.08$ & $3695 \pm 82$ & $0.49 \pm 0.05$ & $0.0384 \pm 0.0090$ & $0.52 \pm 0.07$ & Visible \\
\hline LHS 119 & 0 & $-0.11 \pm 0.08$ & $3523 \pm 73$ & $0.40 \pm 0.05$ & $0.0223 \pm 0.0062$ & $0.41 \pm 0.07$ & $K$-band \\
\hline LHS 1122 & 3143 & $0.01 \pm 0.09$ & $3596 \pm 73$ & $0.44 \pm 0.05$ & $0.0287 \pm 0.0069$ & $0.46 \pm 0.06$ & $K$-band \\
\hline $\mathrm{BD}+61195$ & 4872 & $0.15 \pm 0.08$ & $3835 \pm 73$ & $0.54 \pm 0.04$ & $0.0549 \pm 0.0099$ & $0.58 \pm 0.07$ & $K$-band \\
\hline YZ Cet & 5643 & $-0.26 \pm 0.08$ & $3125 \pm 61$ & $-0.19 \pm 0.00$ & $-0.0033 \pm 0.0000$ & $-0.14 \pm 0.00$ & Visible \\
\hline
\end{tabular}

(This table is available in its entirety in a machine-readable form in the online journal. A portion is shown here for guidance regarding its form and content.)

cross-correlating each spectrum to a template from Bochanski et al. (2007) of the corresponding spectral type. More details on our reduction can be found in Lépine et al. (2013).

We determined the physical parameters of the stars with SNIFS spectra using a modified version of the procedure described in Boyajian et al. (2012) and Mann et al. (2013b). This procedure performs a best-fit match between a stellar spectrum and spectra generated by the PHOENIX stellar atmosphere model (Rajpurohit et al. 2013). A fixed set of wavelength intervals where the observations and model disagree are excluded in such a way as to achieve agreement between best-fit values of effective temperatures $T_{\text {eff }}$ and the bolometric temperatures of calibrator stars established by measuring angular radii and bolometric fluxes (Boyajian et al. 2012; Mann et al. 2013b). We used the BT-SETTL grid of model atmospheres based on the solar abundances of Caffau et al. (2011). We incorporated the $[\mathrm{Fe} / \mathrm{H}]$ determined from our SpeX spectra (Section 2.1) as a constraint on the fit by adding an additional term to $\chi^{2}$. We constructed average spectra from randomly selected sets of three of the best-fit grid points to identify better fits in an interpolated grid. Error in $T_{\text {eff }}$ was determined by adding random noise to each spectrum according to the formal error. We added $60 \mathrm{~K}$ error in quadrature to represent the "floor" in error from the calibration itself.

For stars without visible-wavelength spectra, we estimated $T_{\text {eff }}$ using spectral curvature indices calculated from the SpeX $K$-band spectra and the calibration described in Mann et al. (2013b). Formal errors in the indices were calculated using 100 Monte Carlo realizations; however, the error in $T_{\text {eff }}$ is dominated by the residual $73 \mathrm{~K}$ error in the calibration (Mann et al. 2013b).

We converted values of $T_{\text {eff }}$ into radii, luminosities, and masses using the metallicity-independent empirical relations of Mann et al. (2013b). Errors in these parameters were calculated based on the error in the slope combined with the slope of each empirical curve, plus the error in the calibrations added in quadrature. The coolest star in our calibration has $T_{\text {eff }}=3238 \mathrm{~K}$; the other parameters of RM06 stars with $T_{\text {eff }}$ below this value were assigned upper limits. Values are reported in Table 1.

\section{METALLICITIES AND MASSES OF M DWARFS IN THE SOLAR NEIGHBORHOOD}

The distribution of spectroscopic metallicities of $121 \mathrm{M}$ dwarfs from the RM06 catalog is plotted in Figure 1. The mean and median are $-0.050 \pm 0.008$ and $-0.060 \pm 0.014$, respectively, with uncertainties determined by Monte Carlo

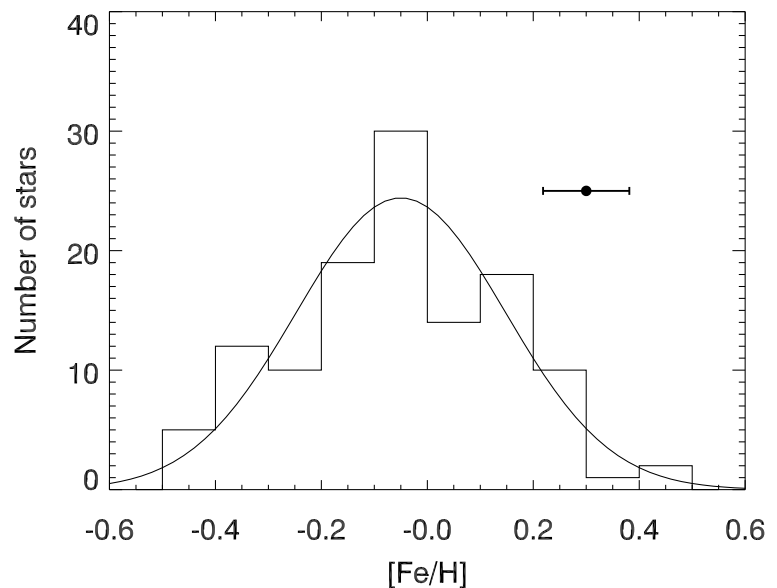

Figure 1. Distribution of $[\mathrm{Fe} / \mathrm{H}]$ for $121 \mathrm{M}$ dwarf stars based on SpeX infrared spectra. The curve is a Gaussian fit to the distribution with a mean of $[\mathrm{Fe} / \mathrm{H}]=$ -0.05 and $\sigma$ of $0.20 \mathrm{dex}$.

simulation. The standard deviation is 0.20 dex and the intrinsic deviation (after subtracting formal errors in quadrature) is 0.18 dex. The distribution is well-described by a Gaussian (Kolmogorov-Smirnov test probability 0.93 that the sample is drawn from the best-fit Gaussian). All of these values are very close to those established using visible-wavelength spectra on a much larger sample of bright nearby $\mathrm{M}$ dwarfs (Gaidos et al. 2014).

There have been other studies of the metallicities of nearby M dwarfs in exoplanet surveys, including Johnson \& Apps (2009), Schlaufman \& Laughlin (2010), and Rojas-Ayala et al. (2010). The most similar study is that of Rojas-Ayala et al. (2012a), which also used infrared spectra and some of the same lines used here. Figure 2 shows excellent agreement between estimates for the 39 stars that overlap with our RM06 sample. The mean difference is statistically insignificant $(0.03 \pm 0.03)$ and the reduced chi-squared $\chi_{v}^{2}$ of 0.23 suggests that our formal errors (and/or theirs) are too conservative.

The largest studies have been those of Neves et al. (2012, 2013) of the CPS sample and southern M dwarfs observed by the ESO/HARPS spectrograph (Bonfils et al. 2013). Neves et al. (2012) used the spectroscopically determined metallicities of primaries in solar-type $M$ dwarf binaries to refine the photometric M dwarf metallicity calibration that Schlaufman \& Laughlin (2010) developed based on $V-K_{s}$ colors and absolute 


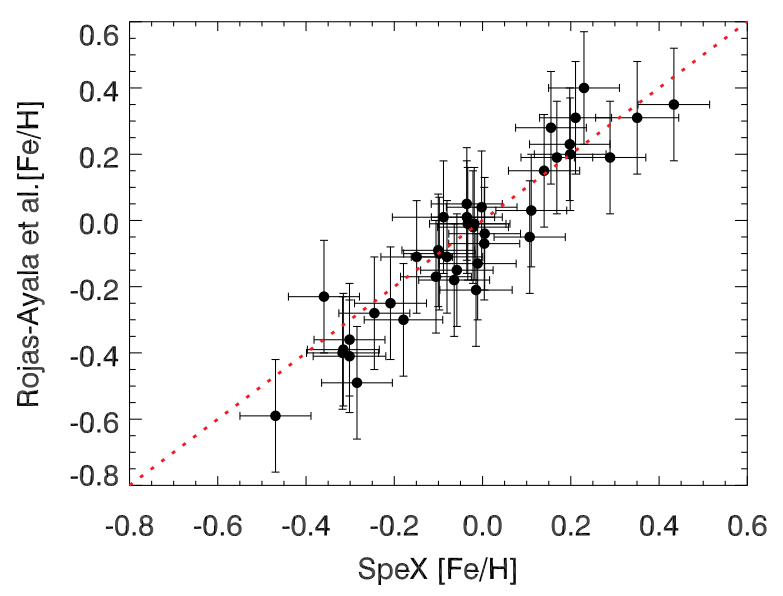

Figure 2. Comparison of $39 \mathrm{M}$ dwarf metallicities based on SpeX infrared spectra (this work) with those of Rojas-Ayala et al. (2012a). The dashed red line is equality. The mean difference is $0.03 \pm 0.03$ dex and the standard deviation is 0.09 dex.

(A color version of this figure is available in the online journal.)

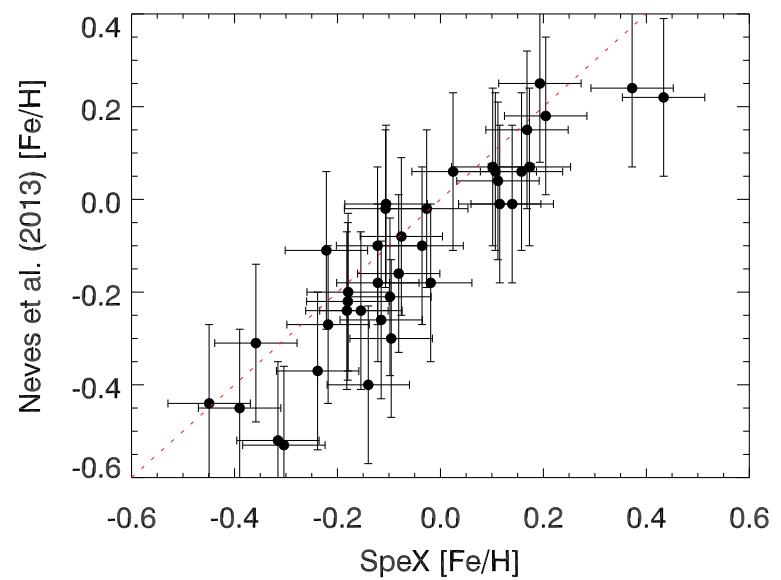

Figure 3. Comparison of $115 \mathrm{M}$ dwarf metallicities based on SpeX infrared spectra (this work) with those based on calibrated photometry (Neves et al. 2012). The dashed red line is equality. The mean difference is $0.08 \pm 0.02 \mathrm{dex}$ and the standard deviation is 0.1 dex.

(A color version of this figure is available in the online journal.)

$K_{s}$ magnitudes. They then applied this to the CPS sample (Neves et al. 2013). They reported a residual dispersion between the spectroscopic values of the solar-type primaries and the photometric values of the $\mathrm{M}$ dwarf secondaries of 0.17 dex, which we adopted as their formal error. Separately, Neves et al. (2013) correlated lines or features in HARPS visible-wavelength echelle spectra with the photometric metallicities of a sample of M dwarfs. They applied this relation to $102 \mathrm{M}$ dwarfs in the HARPS Guaranteed Time Observations (GTO) sample (Bonfils et al. 2013). They gave a calibration dispersion of $0.08 \mathrm{dex}$, and we adopted this as the formal error.

We have metallicities for 115 and 38 stars in the Neves et al. (2012, 2013) samples. Our values are compared with theirs in Figures 3 and 4 . The weighted mean differences (this work: Neves) are $0.08 \pm 0.02$ and $0.06 \pm 0.02$, respectively. The respective standard deviations are 0.10 and 0.08 dex, corresponding to $\chi_{v}^{2}$ values of 0.28 and 0.58 . The small offsets are consistent with the finding by Sousa et al. (2008) that the method of Santos et al. (2004) to determine the metallicities of the primary stars used by Neves et al. (2012) is on the same scale as the SPOCS catalog, and that the spectroscopic scale

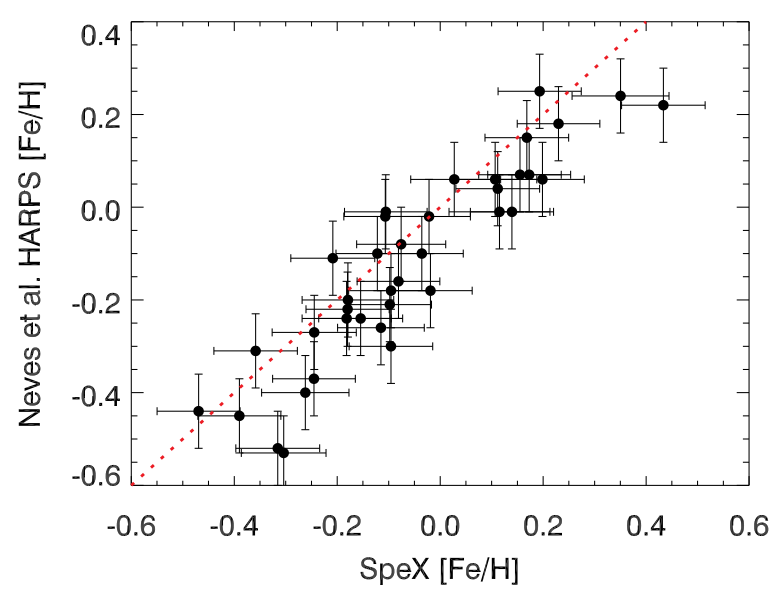

Figure 4. Comparison of $38 \mathrm{M}$ dwarf metallicities based on SpeX infrared spectra (this work) with those based on HARPS visible-wavelength spectra (Neves et al. 2013). The dashed red line is equality. The mean difference is $0.06 \pm 0.02$ dex and the standard deviation is 0.09 dex

(A color version of this figure is available in the online journal.)

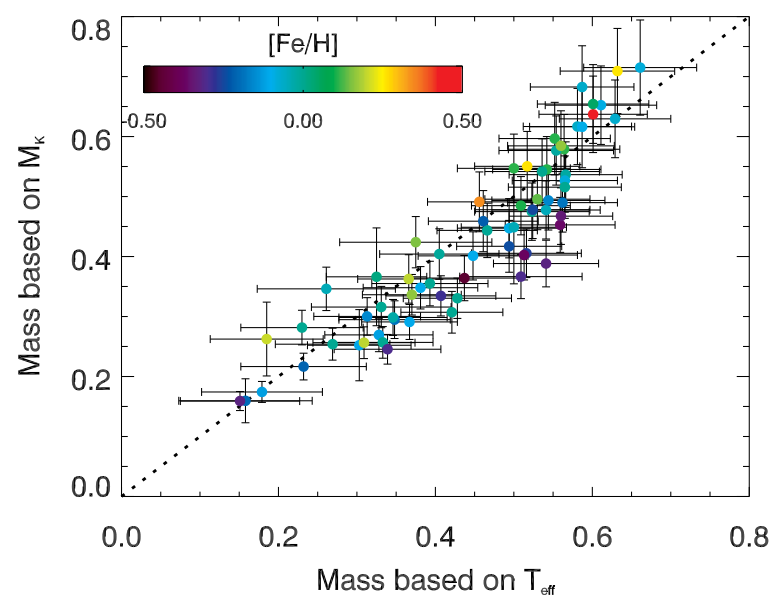

Figure 5. Comparison of estimated mass of $M$ dwarfs based on spectroscopic estimation of $T_{\text {eff }}$ vs. those based on absolute $K_{S}$-band magnitudes and the mass-luminosity relation of Delfosse et al. (2000). Points are colored according to metallicity. The dashed line is equality. The mean of the ratio is 1.07 , and the standard deviation is 0.15 .

(A color version of this figure is available in the online journal.)

of Neves et al. (2013) is tied to the photometric calibration of Neves et al. (2012). In addition, there is significant overlap in the solar-type $\mathrm{M}$ dwarf binary calibrators used by Neves et al. (2013) and Mann et al. (2013a). The low $\chi_{v}^{2}$ values may indicate that errors are overestimated.

Of the 121 stars in our sample, 118 have parallaxes. We estimated masses using the $M_{K}$-mass relation in Delfosse et al. (2000) and compared these with values based on our $T_{\text {eff }}$ estimates (Section 2.2) and the $T_{\text {eff }}$-mass relation in Mann et al. (2013b). (That relation in turn is based on masses of calibrator M dwarfs from Boyajian et al. 2012 using the mass-luminosity relation of Henry \& McCarthy 1993). There is good agreement between the estimates (Figure 5); the mean ratio of the $T_{\text {eff }}$-based over the $M_{K}$-based estimates is 1.07 , the standard deviation is 0.15 , and $\chi_{v}^{2}$ is 0.86 . There is a possible trend with metallicity, with the temperatures (and thus masses) of metal-poor stars based on $T_{\text {eff }}$ estimates exceeding the estimates based on $M_{K}$ and Delfosse et al. (2000). This plot does not establish which method is effected by this systematic; if it exists, however, a plot of $T_{\text {eff }}$ from fits to PHOENIX models versus values 


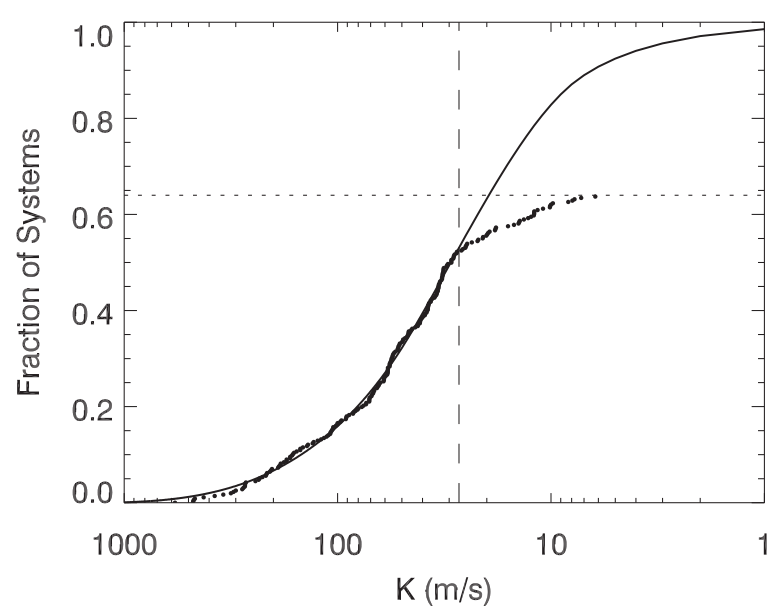

Figure 6. Cumulative distribution of the RV amplitude $K$ of all Doppler-detected giant planets with $0.1 M_{J}<M \sin i<10 M_{J}$ and $P<2$ yr in the Exoplanet Orbit Database (Wright et al. 2011b). The vertical dashed line at $K=37 \mathrm{~m} \mathrm{~s}^{-1}$ marks a break in the distribution we interpret to indicate the onset of detection incompleteness. The solid curve is a best-fit for planets with a power-law massdistribution index of 0.78 over $0.3-10 M_{J}$, a flat distribution with log orbital period over $2 \mathrm{~d}<P<2 \mathrm{yr}$, a Rayleigh-distributed eccentricity distribution with mean $\bar{e}=0.25$, and isotropically inclined orbits.

derived from the curvature of the $K$-band spectrum (Mann et al. 2013 b) show no trend with $[\mathrm{Fe} / \mathrm{H}]$. The $M_{K}$-based estimates have slightly smaller formal errors, and we use them in our subsequent analysis. For the three stars without parallaxes or visible-wavelength spectra, we used the $K$-band-based masses.

\section{ANALYSIS: PLANETS, MASSES, AND METALLICITIES}

We compared metallicities and giant planet occurrence in the M dwarf sample to that of the SPOCS sample (Valenti \& Fischer 2005). Critically, and as discussed in Section 2, our $\mathrm{M}$ dwarf metallicities are calibrated against solar-type primaries that have been analyzed in an identical manner to SPOCS (Mann et al. 2013a) and for which the offset of values for overlapping SPOCS and calibrator stars is $<0.01 \mathrm{dex}$. In fact, some of the calibrator primaries are SPOCS stars. Thus, any systematic error in SPOCS metallicities is also present in our $M$ dwarf metallicities and to zero order is removed. Because our $\mathrm{M}$ dwarf calibration was performed over a range of $[\mathrm{Fe} / \mathrm{H}]$ similar to that of SPOCS, it is removed to at least first order in $[\mathrm{Fe} / \mathrm{H}]$ as well. The median and mean $[\mathrm{Fe} / \mathrm{H}]$ of SPOCS stars are +0.04 and -0.01 , respectively and, thus, are on average slightly more metal-rich than the statistics of our RM06 M dwarf sample.

We obtained current planet data from the Exoplanet Orbit Database (Wright et al. 2011b). For consistency with previous analyses, we consider giant planets with projected masses $0.1 M_{J}<M \sin i<10 M_{J}$ and orbital periods $P<$ 2 yr. The lower mass limit removes Neptune-size planets that are considered a distinct population of objects with a different formation pathway (Benz et al. 2014). We estimated the completeness of the exoplanet archive catalog with RV amplitude $K$ from the cumulative distribution of detected planets itself (Figure 6). We identified the break in the distribution at $K \approx 27 \mathrm{~m} \mathrm{~s}^{-1}$ as the point below which the catalog becomes incomplete for the planet mass and period range of interest. A planet with $M \sin i=1 M_{J}$ on a two year circular orbit around a solar-mass star produces $K \approx 27 \mathrm{~m} \mathrm{~s}^{-1}$, a signal that can be readily detected in high-precision Doppler surveys, provided there is a sufficient time baseline. To calculate the completeness of the catalog over some actual mass range, accounting for the underlying distribution of eccentricities and orbital inclinations, we assume that the distribution with $M$ and $P$ are smooth functions of the usual form:

$$
d N=M^{-\alpha} P^{-\beta} d \log M d \log P .
$$

We assumed a Rayleigh distribution for orbital eccentricities with a mean $\bar{e}$ (Moorhead et al. 2011) and isotropically distributed orbital inclinations. We determined the completeness $C$ of the catalog by calculating the cumulative distribution of $K$ for a population of Saturn- to super-Jupiter-masses $\left(0.3 M_{J}<M<10 M_{J}\right)$ and periods 3 day $<P<2 \mathrm{yr}$, and finding best-fit values of $\alpha, \beta, \bar{e}$, and $C$ by nonlinear leastsquares fitting with the MPFIT routine (Markwardt 2009). We find $\alpha=0.78, \beta=-0.003, \bar{e}=0.25$, consistent with previous findings (Cumming et al. 2008), and $C=0.64$. Thus, our adopted subsample of the catalog captures the majority of Saturn-mass and larger objects to two year periods. We use a single value of $C$ to describe our completeness.

There are 73 giant planets satisfying our criteria among 1039 SPOCS stars. We identified giant planets orbiting three RM06 stars for which we have determined [Fe/H]: GJ 1148, GJ 649, and GJ 876, with metallicities of $-0.04 \pm 0.08,0.00 \pm 0.08$, and $+0.17 \pm 0.08$, respectively. Rojas-Ayala et al. (2012a) found metallicities of $+0.05 \pm 0.17,-0.04 \pm 0.17$, and $+0.19 \pm 0.17$, respectively, in excellent agreement with our values. Likewise, Neves et al. (2013) found metallicities of $+0.07,-0.08$, and +0.12 .

We infer a planet-metallicity relation of the standard form

$$
f([\mathrm{Fe} / \mathrm{H}])=f_{0} 10^{a[\mathrm{Fe} / \mathrm{H}]}
$$

and determine the values of $f_{0}$ and $a$ that maximize the binomial likelihood, i.e.,

$$
\log \mathcal{L}=\sum_{i}^{D} \log f\left([\mathrm{Fe} / \mathrm{H}]_{\mathrm{i}}\right)+\sum_{j}^{\mathrm{ND}} \log \left[1-C f\left([\mathrm{Fe} / \mathrm{H}]_{\mathrm{j}}\right)\right]
$$

where the first and second summations are over $D$ and ND systems with and without detected giant planets, respectively. (The factor $C$ in the first summed logarithms contributes only a constant term to the likelihood and is thus omitted.) For the SPOCS stars, we found $f_{0}=0.070$ and $a=1.80$, consistent with Fischer \& Valenti (2005). Our value of $f_{0}$ is slightly lower than Cumming et al. (2008) when extrapolated to $P=2000$ days. Figure 7 shows that Equation (2) with these parameter values describes the planet-metallicity distribution well. The notable exception is HIP 37124, which has $[\mathrm{Fe} / \mathrm{H}]=$ -0.44 and three close-in giant planets with a possible 2:1 meanmotion resonance (Wright et al. 2011a). The metal-poor nature of this star has been confirmed by independent observations (Santos et al. 2003; Kang et al. 2011).

We test the null hypothesis, i.e., that the planet-metallicity relation derived for the SPOCS sample adequately describes giant planet occurrence among $\mathrm{M}$ dwarfs. The expected number of giant planets using our values of $[\mathrm{Fe} / \mathrm{H}]$ and Equation (2) is 6.4. Assuming Poisson statistics, the probabilities of finding three or fewer planets is 0.12 . Since the equivalent of $1 \sigma$ probability is one half of $1-0.68$, or 0.16 , we conclude that the occurrence of giant planets around $\mathrm{M}$ dwarfs differs from that of solar-type stars at slightly more than $1 \sigma$ significance. Without a correction for the metallicity distributions of the SPOCS and 


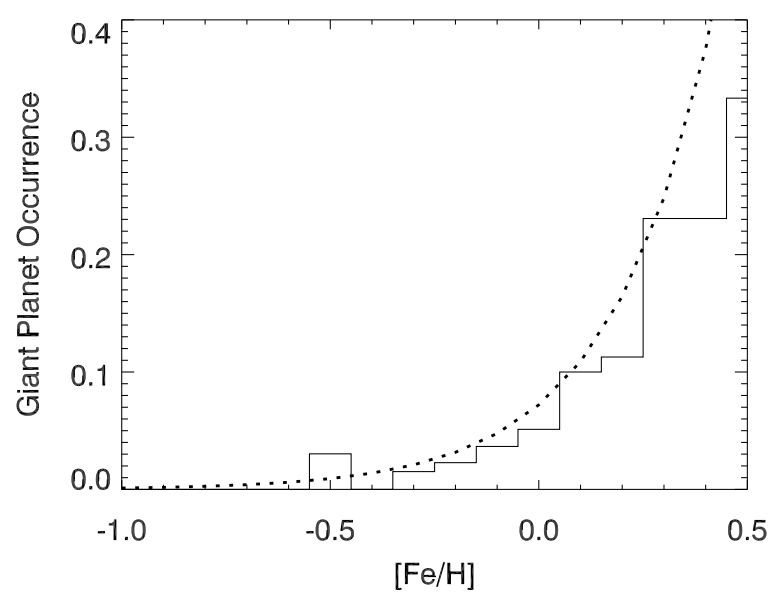

Figure 7. Occurrence of giant planets $\left(0.1 M_{J}<M \sin i<10 M_{J}, P<2 \mathrm{yr}\right)$ among SPOCS stars vs. metallicity. The dashed line is Equation (2) with maximum likelihood coefficients 1.80 dex per dex and an occurrence of 0.070 at solar metallicity, and a completeness of 0.651 .

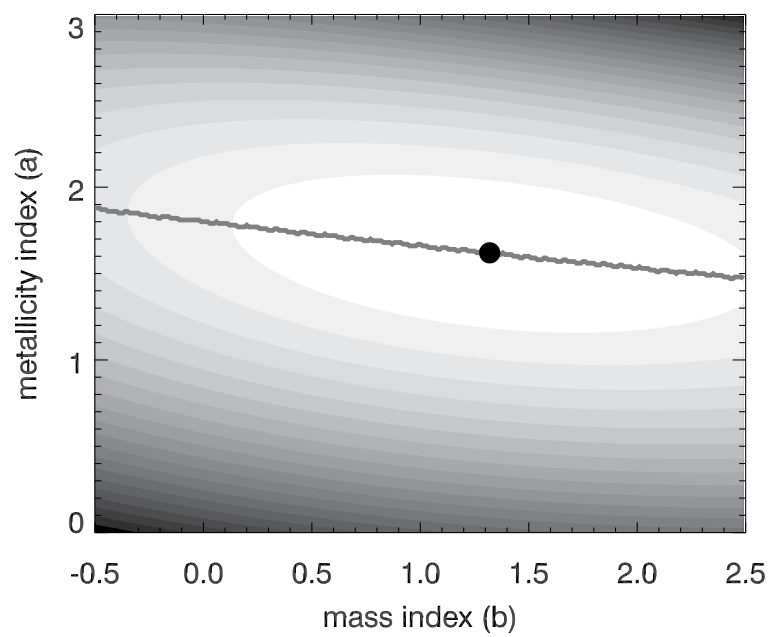

Figure 8. Contour plot of log likelihood vs. metallicity and mass occurrence parameters $a$ and $b$ among SPOCS solar-type stars. Each shade represents one relative unit of $\log$ likelihood, with lighter shades representing higher values. The black dot is the location of the maximum $(a=1.62, b=1.32)$ and the gray line is a "ridge" of values of $a$ that maximize the likelihood for a given $b$.

M dwarf samples we would expect 8.5 planets and the difference would be much more significant.

Although dependence on stellar mass is not required to explain the observations, what mass dependence can be excluded? We assumed the form

$$
f=f_{0} 10^{a[\mathrm{Fe} / \mathrm{H}]} M_{*}^{b},
$$

where $M_{*}$ is in solar units and we consider $b \in[-1,2]$. For SPOCS stars we used the mass estimates from Valenti \& Fischer (2005). As discussed above, SPOCS mass assignments are controversial for higher-mass, evolved stars (Lloyd 2013), and they are probably not reliable for late $\mathrm{K}$ and $\mathrm{M}$ dwarf stars. Therefore, we only considered the 815 stars (55 planets) with $0.7 M_{\odot}<M_{*}<1.5 M_{\odot}$ and $\log g>3.85$.

Figure 8 is a contour of the relative log likelihood versus $a$ and $b$ for SPOCS stars. Regions of parameter space with high or low values of $a$ are definitively excluded, but a wide range of $b$ is allowed. The maximum is at $a \approx 1.62$ and $b \approx 1.32$ (black dot), in agreement with Montet et al. (2014). The gray line is

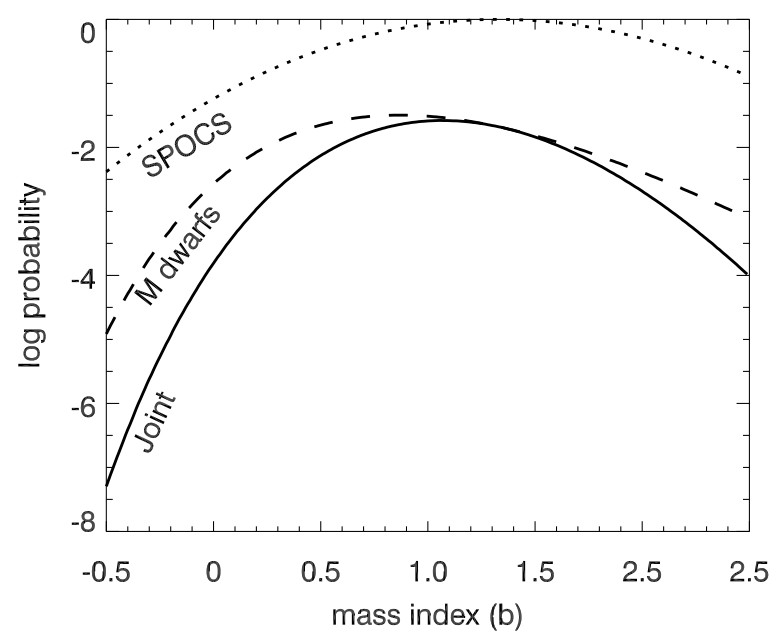

Figure 9. Log likelihood relative to the maximum value for SPOCS stars (dashed line), logarithm of the probability of the observed number of giant planets in the RM06 M dwarf sample (dotted line), and the joint likelihoods (solid line) vs. mass index $b$ and metallicity index $a$ along the trajectory in Figure 8.

the "ridge" of values of $a$ maximizing the likelihood for a given value of $b$. Figure 9 plots the relative log likelihood along the "ridge" (dotted line). Also plotted is the probability of observing three planets in our $\mathrm{M}$ dwarf sample along this trajectory in $a-b$ parameter space (dashed line). This is maximized at $b=0.87$, consistent with the finding of Montet et al. (2014). The solid line is the joint likelihood, assuming that a single mass-metallicity-planet relation applies to all stars, and it peaks at $b=1.06$. Assuming asymptotic normality (AN, parabolic behavior of the logarithmic likelihood near its maximum) for the joint likelihood curve, the uncertainty in $b$ is \pm 0.47 . Thus, the data support a roughly linear stellar mass dependence but with weak significance and any scenario between no mass dependence $(b \sim 0)$ and strong mass dependence $(b \sim 2)$ cannot be excluded. If we include the stars in the SPOCS sample with $M_{*}>1.5 M_{\odot}$ and accept those mass estimates, then we find $b=0.37 \pm 0.32$; and significant mass dependence is excluded because there are too few planets around the most massive stars. Clearly, reliable mass estimates for these stars would be very valuable.

Finally, we independently evaluated the giant-planetmetallicity relation of $\mathrm{M}$ dwarfs and assessed whether it is statistically distinguishable from that of SPOCS solar-type stars. An erroneous assumption of a universal relation could mask mass dependence. For example, if the metallicity relation for $\mathrm{M}$ dwarfs were less steep than for solar-type stars, a greater mass dependence would be required to explain the relative deficit of giant planets. We estimated uncertainties using the assumption of AN for the likelihood curve as well as by constructing 100 Monte Carlo representations of the data assuming normally distributed errors in metallicity; for the SPOCS stars, we assume an error of 0.03 dex for all stars (Valenti \& Fischer 2005). For SPOCS stars, we found $a=1.80$ with an uncertainty of \pm 0.05 based on Monte Carlo simulations, but 0.31 based on AN. For the M dwarfs, we found $a=1.06$ with uncertainties of 0.42 based on Monte Carlo and \pm 1.25 based on AN. (The best-fit $f_{0}$ is 0.025 .) The relative magnitudes of the uncertainties indicates that it is sample size, rather than metallicity precision, which limits statistical significance. The difference between values of $a$ for SPOCS stars and the M dwarfs is less than $1 \sigma$ and not significant (see Section 5). 


\section{DISCUSSION AND CONCLUSIONS}

We obtained infrared and visible-wavelength spectra to estimate the metallicities and other fundamental parameters of nearby M dwarfs monitored by the CPS Doppler exoplanet survey. These $\mathrm{M}$ dwarfs are on average slightly more metal poor (0.1 dex) than the SPOCS comparison catalog of solar-mass stars that has also been monitored for planets. We then determined the number of Doppler-detected giant planets $(M \sin i=$ $0.1-10 M_{J}$ ) with orbital periods $<2 \mathrm{yr}$ around the $\mathrm{M}$ dwarfs as well as the solar-type stars.

Heavy, or metal? When we assumed that giant planet occurrence depends only on metallicity, we derived a planet-metallicity relation for solar-type stars (exponent $a=$ 1.80 dex per dex) that overpredicts occurrence around M dwarfs, but the discrepancy is not statistically significant. On the other hand, when we allowed both mass and metallicity dependence, we found mass dependence of $b \sim 1$ dex per dex for both solarmass and M dwarf stars, but this is only marginally significant and the hypothesis of no mass dependence cannot be excluded. We find a metallicity dependence for $\mathrm{M}$ dwarfs that is lower than that of solar-mass stars ( $a \approx 1$ rather than $a \approx 1.8$ ), but this difference is, yet again, not significant due to the small size of the $\mathrm{M}$ dwarf sample.

Because our M dwarf metallicities are calibrated against the metallicities of primaries determined using the same technique as the SPOCS catalog (and in fact, some of the calibrators are in the SPOCS catalog), comparisons between the two samples are immune to any systematic error in the masses and metallicities of SPOCS stars. Nevertheless, the known covariance between estimates of $[\mathrm{Fe} / \mathrm{H}]$ and $T_{\text {eff }}$ (and hence mass) from SME (Torres et al. 2012) still effects our estimates of metallicity and mass indices $a$ and $b$. Independent estimation of the $T_{\text {eff }}$ of SPOCS stars using the spectroscopic, model-independent methods described in Mann et al. (2013b), which in turn are based on the calibration stars studied by Boyajian et al. (2012), and then revision of $[\mathrm{Fe} / \mathrm{H}]$ based on those $T_{\text {eff }}$ values are warranted.

In contrast with Johnson et al. (2010), only one of the three giant planet hosts in our $\mathrm{M}$ dwarf sample is metal-rich with respect to the sample mean. Our values agree with previous estimates for these stars based on infrared spectra (Rojas-Ayala et al. 2012b) or calibrated photometry (Neves et al. 2013). Curiously, if we relax our orbital period restriction, two more giant planet hosts enter our sample: GJ 179b (6.26 yr orbit) and GJ $849 \mathrm{~b}$ (5.16 yr orbit). Both of these are metal-rich, but two objects do not make a trend.

Our $\mathrm{M}$ dwarf planet-metallicity relation is more shallow $(a=1.05)$ than that found by others $(a \approx 2-3$; Johnson et al. 2010; Neves et al. 2013; Montet et al. 2014). The discrepancy with Neves et al. (2013) is particularly puzzling since there is excellent agreement between the two sets of metallicity estimates (Figure 3). Some part of the difference could be explained by the 0.08 dex offset in mean $[\mathrm{Fe} / \mathrm{H}]$, but also the exact distribution of metallicities may matter. As an experiment, we substituted 115 values for $[\mathrm{Fe} / \mathrm{H}]$ from Neves et al. (2013) into our analysis and found $a=1.99$, almost exactly the value of 1.97 found by Neves et al. (2013). Thus, the discrepancy is not due to any methodological difference but instead arises from the sensitivity of maximum-likelihood estimates of $a$ to the exact distribution with $[\mathrm{Fe} / \mathrm{H}]$. This is also suggested by the large AN-based uncertainties in $a$. Support for a lower value of $a$ also comes from microlensing surveys of the metal-rich Galactic
Bulge, which find an order of magnitude fewer giant planets around $\mathrm{M}$ dwarfs than predicted by a steep planet-metallicity dependence (Clanton \& Gaudi 2014).

To explore the effects of sample size and measurement error, we carried out Monte Carlo simulations of the recovery of $a$ from synthetic populations of stars and planets. Each simulated a sample of 121 stars to which random Gaussian-distributed metallicities were assigned. Planets were placed around stars with a probability given by Equation (2) and fixed value of $a=$ 2. Then, Gaussian-distributed measurement errors were added to the actual metallicities, and maximum-likelihood values of $\alpha$ were recovered using Equation (3). In the absence of measurement error, the mean of the inferred values of $a$ was close to the actual value, but the standard deviation was $\sigma \approx 1$. The large value of $\sigma$ agrees with our AN-based estimates of the uncertainty in $a$. The introduction of measurement errors lowers the inferred value of $a$ relative to the actual value. For example, if the observed dispersion of 0.20 dex is the product of an intrinsic variation of $0.18 \mathrm{dex}$ and measurement error equal to 0.08 dex then the mean value inferred from $\alpha=2$ simulations is $\alpha=1.85$. The larger the contribution of error to the dispersion, the greater the difference. This effect alone, however, cannot explain the difference between our results and Neves et al. (2013) because our errors are similar in magnitude.

At orbital periods $\gg 2$ yr Doppler surveys become very incomplete, i.e., missing the lower-mass $\left(\lesssim 1 M_{J}\right)$ majority of the giant planet population. This can be partially ameliorated if long-term trends are included (Montet et al. 2014). On the other hand, microlensing surveys can already detect such planets, albeit around a population of distant $\mathrm{M}$ dwarfs in the direction of the Galactic Bulge. Clanton \& Gaudi (2014) performed a joint analysis of RV and microlensing surveys and estimated that $15 \% \pm 6 \%$ of $\mathrm{M}$ dwarfs host planets with $M \sin i>0.1 M_{J}$ and $P<27$ yr. However, two-thirds of these orbits were outside $2.7 \mathrm{yr}$, demanding a marked departure from a flat $\log P$ distribution.

Kepler weighs in. The NASA Kepler mission could readily detect giant planets around both solar-type stars and M dwarfs; but, the former lie at kiloparsec distances and their metallicities may not follow the distribution in the solar neighborhood. Moreover, while the $[\mathrm{Fe} / \mathrm{H}]$ of some individual solar-type host stars has been determined, the underlying metallicity distribution has not yet been established. In contrast, Kepler $\mathrm{M}$ dwarfs are a factor of 10 closer and are likely to resemble their nearby counterparts (Gaidos et al. 2012). Mann et al. (2013c) found a median $[\mathrm{Fe} / \mathrm{H}]$ of $-0.10 \pm 0.03$ among non-KOI hosts, only slightly more metal poor than the RM06 sample. We calculated the expected occurrence of giant planets around Kepler $\mathrm{M}$ dwarfs based on the SPOCS distribution and found it to be 0.071. Adjusting to $2<P<365$ days assuming a flat distribution with $\log P$, the predicted occurrence is 0.06 .

We determined the observed occurrence of giant planets $\left(R_{p}>6 R_{\oplus}\right)$ around 3828 Kepler stars with $2800 \mathrm{~K}<T_{\text {eff }}<$ $4200 \mathrm{~K}$ and $\log g>4.2$; stellar parameters that were estimated by Bayesian analysis of photometry in the Kepler Input Catalog (Brown et al. 2011); the Dartmouth stellar evolution models (Dotter et al. 2008); and priors on stellar, metallicity, age, mass, and distance (Gaidos 2013). We restricted the sample to orbits with a period $P<1 \mathrm{yr}$ and assumed, based on the large transit depths $(>1 \%)$, that all giant planets that transit would be detected. We assumed Rayleigh-distributed eccentricities with a mean of 0.272 (see above) and calculated the mean transit probability $p$ for each star assuming a flat 
logarithmic distribution with $P$. We then found the occurrence that maximized the binomial log likelihood

$$
\ln \ell=N_{D} \ln f+\sum_{i}^{\mathrm{ND}}\left(1-f p_{i}\right),
$$

where $N_{D}$ is the number of detections, and the sum is over all stars without detections. We identified two candidate giant planets and found that the likelihood is maximized at $f=0.036$ with an uncertainty of \pm 0.021 based on AN. This is additionally tantalizing but not significant $(1.1 \sigma)$ evidence for a deficit of giant planets around $\mathrm{M}$ dwarfs relative to solar-type stars.

Is theory in irons? Naively, if the formation of giant planets depends on the mass of solids in a protoplanetary disk, and this scales with both stellar mass and metallicity, then one would expect that the planet-metallicity relation for $\mathrm{M}$ dwarfs would be steeper than that of solar-mass stars. While our analysis cannot exclude similar values of $a$ for M dwarfs and SPOCS stars, they seem to exclude $a \gtrsim 3$, as does the microlensing analysis of Clanton \& Gaudi (2014). Perhaps it is not the mass of solids but some other factor, such as disk lifetime, that regulates giant planet formation (Yasui et al. 2009; Owen et al. 2012). This is certainly suggested by the apparent absence of a planet-metallicity relation for Neptune-size planets that could serve as the cores of giant planets (Neves et al. 2013; Mann et al. 2013c).

This analysis, and essentially all others, is predicated on simple and universal relationships between planet occurrence and stellar mass/metallicity. Nature may not be so compliant. Orbital migration is now widely accepted to play an important role in determining the distribution of giant planets, particularly those close to their host stars and hence those that are overrepresented in Doppler and transit surveys. However, many aspects of that migration are not well understood, including the particularly salient aspect of what halts it (Hasegawa \& Ida 2013). Analysis of microlensing surveys suggest that the majority of giant planets orbiting $M$ dwarfs are found beyond $\sim 1.5 \mathrm{AU}$; these must be considered and it is hoped that they include Montet et al. (2014) when elucidating the planet-metallicity relationship of such stars.

In our analysis, we have assumed that the mass distribution (Equation (1)) of giant planets orbiting M dwarfs is the same as that around solar-type stars. If $\mathrm{M}$ dwarfs have less massive disks or if the gas dissipates more rapidly, giant planet formation would terminate at lower masses, producing a steeper mass distribution with a larger value of $\alpha$, a possibility suggested by Neves et al. (2013). Doppler surveys, which are biased with mass, would then find an apparent deficit of giant planets around $\mathrm{M}$ dwarfs relative to solar-type stars, even if the actual occurrence is the same.

Massive improvements ahead. Our analysis, along with every previous one, is limited by the comparatively small number of $\mathrm{M}$ dwarfs in exoplanet surveys. A modest but important step will be spectroscopic measurement of $[\mathrm{Fe} / \mathrm{H}]$ in all $\mathrm{M}$ dwarf stars in Doppler RV surveys, especially the HARPS GTO survey (Bonfils et al. 2013). However, this will not even double the size of the sample described here. The advent of high-precision spectrographs operating at infrared wavelengths where there is considerably more signal from $\mathrm{M}$ dwarfs will usher in larger Doppler RV surveys (Tamura et al. 2012; Thibault et al. 2012; Quirrenbach et al. 2012; Mahadevan et al. 2012). These surveys are typically envisioned to include $\sim 300$ stars, and proper coordination could lead to an order-of-magnitude improvement in the sample size. An important consideration is the time baseline needed to detect planets over the orbital period range considered here, which is well beyond the M dwarf "habitable zone" whose compact size drives the design of these surveys. An alternative strategy is to survey the most metal-rich $\mathrm{M}$ dwarfs with a Doppler survey, thus taking advantage of a longer "lever arm" with which to constrain the mass-metallicity index $a$. We are pursuing such a survey at visible and, in the future, infrared wavelengths.

The Kepler-2 mission (Howell et al. 2014) and NASA Transiting Exoplanet Survey Satellite mission (Ricker et al. 2014) will make modest contributions because of the short observation periods $\ll 1 \mathrm{yr}$ and the strong bias of the transit method toward short-period orbits where giant planets are rare around M dwarfs. In contrast, the Gaia spacecraft will monitor thousands of nearby $\mathrm{M}$ dwarfs with an astrometric precision of tens of $\mu$ arcsec (de Bruijne 2012). Sozzetti et al. (2014) estimated that $\sim 100$ giant planets would be detected around the nearest $\mathrm{M}$ dwarfs $(d \lesssim 50 \mathrm{pc})$. Conveniently, Gaia will also provide precise parallaxes with which $M_{K}$ and, hence, mass can be estimated. Follow-up spectroscopy of M dwarfs with Gaiadetected companions and/or the calibration of Gaia spectra of the Ca II infrared triplet to determine metallicity should be a high priority.

This research was supported by NASA grants NNX10AQ36G and NNX11AC33G to E.G. It has made use of the Exoplanet Orbit Database and the Exoplanet Data Explorer at exoplanets.org.

\section{REFERENCES}

Aldering, G., Adam, G., Antilogus, P., et al. 2002, Proc. SPIE, 4836, 61

Aldering, G., Antilogus, P., Bailey, S., et al. 2006, ApJ, 650, 510

Andrews, S. M., Rosenfeld, K. A., Kraus, A. L., \& Wilner, D. J. 2013, ApJ, 771,129

Bacon, R., Copin, Y., Monnet, G., et al. 2001, MNRAS, 326, 23

Benz, W., Ida, S., Alibert, Y., Lin, D. N. C., \& Mordasini, C. 2014, arXiv: 1402.7086

Bochanski, J. J., West, A. A., Hawley, S. L., \& Covey, K. R. 2007, AJ, 133, 531

Bohlin, R. C., Dickinson, M. E., \& Calzetti, D. 2001, AJ, 122, 2118

Bonfils, X., Delfosse, X., Udry, S., et al. 2013, A\&A, 549, A109

Boyajian, T. S., von Braun, K., van Belle, G., et al. 2012, ApJ, 757, 112

Brown, T. M., Latham, D. W. D., Everett, M. E. M., \& Esquerdo, G. G. A. 2011, AJ, 142, 112

Buton, C., Copin, Y., Aldering, G., et al. 2013, A\&A, 549, A8

Caffau, E., Ludwig, H.-G., Steffen, M., Freytag, B., \& Bonifacio, P. 2011, SoPh, 268,255

Clanton, C., \& Gaudi, S. 2014, arXiv:1404.7500

Cumming, A., Butler, R. P., Marcy, G. W., et al. 2008, PASP, 120, 531

Cushing, M. C., Rayner, J. T., \& Vacca, W. D. 2005, ApJ, 623, 1115

Cushing, M. C., Vacca, W. D., \& Rayner, J. T. 2004, PASP, 116, 362

de Bruijne, J. H. J. 2012, Ap\&SS, 341, 31

Delfosse, X., Forveille, T., Ségransan, D., et al. 2000, A\&A, 364, 217

Dotter, A., Chaboyer, B., Jevremović, D., et al. 2008, ApJS, 178, 89

Fischer, D. A., \& Valenti, J. 2005, ApJ, 622, 1102

Gaidos, E. 2013, ApJ, 770, 90

Gaidos, E., Fischer, D. A., Mann, A. W., \& Howard, A. W. 2013, ApJ, 771,18

Gaidos, E., Fischer, D. A., Mann, A. W., \& Lépine, S. 2012, ApJ, 746, 36

Gaidos, E., Mann, A. W., Lépine, S., et al. 2014, arXiv:1406.7353

Gonzalez, G. 1998, A\&A, 334, 221

Hamuy, M., Suntzeff, N. B., Heathcote, S. R., et al. 1994, PASP, 106, 566

Hasegawa, Y., \& Ida, S. 2013, ApJ, 774, 146

Helled, R., Bodenheimer, P., Podolak, M., et al. 2013, arXiv:1311.1142

Henry, T. J., \& McCarthy, D. W., Jr. 1993, AJ, 106, 773

Howell, S. B., Sobeck, C., Haas, M., et al. 2014, PASP, 126, 398

Johnson, J. A., Aller, K. M., Howard, A. W., \& Crepp, J. R. 2010, PASP, 122,905

Johnson, J. A., \& Apps, K. 2009, ApJ, 699, 933 
Johnson, J. A., Morton, T. D., \& Wright, J. T. 2013, ApJ, 763, 53

Kang, W., Lee, S.-G., \& Kim, K.-M. 2011, ApJ, 736, 87

Lantz, B., Aldering, G., Antilogus, P., et al. 2004, Proc. SPIE, 5249, 146

Lépine, S., \& Gaidos, E. 2011, AJ, 142, 138

Lépine, S., Hilton, E. J., Mann, A. W., et al. 2013, AJ, 145, 102

Lloyd, J. P. 2011, ApJL, 739, L49

Lloyd, J. P. 2013, ApJL, 774, L2

Mahadevan, S., Ramsey, L., Bender, C., et al. 2012, Proc. SPIE, 8446

Mann, A. W., Brewer, J. M., Gaidos, E., Lépine, S., \& Hilton, E. J. 2013a, AJ, 145,52

Mann, A. W., Gaidos, E., \& Ansdell, M. 2013b, ApJ, 779, 188

Mann, A. W., Gaidos, E., Kraus, A., \& Hilton, E. J. 2013c, ApJ, 770, 43

Markwardt, C. B. 2009, in ASP Conf. Ser. 411, Astronomical Data Analysis Software and Systems XVIII, ed. D. A. Bohlender, D. Durand, \& P. Dowler (San Francisco, CA: ASP), 251

Montet, B. T., Crepp, J. R., Johnson, J. A., Howard, A. W., \& Marcy, G. W. 2014, ApJ, 781, 28

Moorhead, A. V., Ford, E. B., Morehead, R. C., et al. 2011, ApJS, 197, 1

Neves, V., Bonfils, X., Santos, N. C., et al. 2013, A\&A, 551, A36

Neves, V., Bonfils, X., Santos, N. C., et al. 2012, A\&A, 538, A25

Oke, J. B. 1990, AJ, 99, 1621

Önehag, A., Heiter, U., Gustafsson, B., et al. 2012, A\&A, 542, A33

Owen, J. E., Clarke, C. J., \& Ercolano, B. 2012, MNRAS, 422, 1880

Pineda, J. S., Bottom, M., \& Johnson, J. A. 2013, ApJ, 767, 28

Quirrenbach, A., Amado, P. J., Seifert, W., et al. 2012, Proc., SPIE, 8446

Rajpurohit, A. S., Reyle, C., Allard, F., et al. 2013, A\&A, 556, A15
Rauscher, E., \& Marcy, G. W. 2006, PASP, 118, 617

Rayner, J. T., Cushing, M. C., \& Vacca, W. D. 2009, ApJS, 185, 289

Rayner, J. T., Toomey, D. W., Onaka, P. M., et al. 2003, PASP, 115, 362

Ricker, G. R., Winn, J. N., Vanderspek, R., et al. 2014, arXiv:1406.0151

Rojas-Ayala, B., Covey, K., Muirhead, P., \& Lloyd, J. P. 2012a, ApJ, 748, 93

Rojas-Ayala, B., Covey, K. R., Muirhead, P. S., \& Lloyd, J. P. 2010, ApJL, 720, L113

Rojas-Ayala, B., Covey, K. R., Muirhead, P. S., \& Lloyd, J. P. 2012b, ApJ 748, 93

Santos, N. C., Israelian, G., \& Mayor, M. 2004, A\&A, 415, 1153

Santos, N. C., Israelian, G., Mayor, M., Rebolo, R., \& Udry, S. 2003, A\&A, 398,363

Schlaufman, K. C., \& Laughlin, G. 2010, A\&A, 519, A105

Schlaufman, K. C., \& Winn, J. N. 2013, ApJ, 772, 143

Sousa, S. G., Santos, N. C., Mayor, M., et al. 2008, A\&A, 487, 373

Sozzetti, A., Giacobbe, P., Lattanzi, M. G., et al. 2014, MNRAS, 437, 497

Tamura, M., Suto, H., Nishikawa, J., et al. 2012, Proc., SPIE, 8446

Terrien, R. C., Mahadevan, S., Bender, C. F., et al. 2012, ApJL, 747, L38

Thibault, S., Rabou, P., Donati, J.-F., et al. 2012, Proc., SPIE, 8446

Torres, G., Fischer, D. A., Sozzetti, A., et al. 2012, ApJ, 757, 161

Vacca, W. D., Cushing, M. C., \& Rayner, J. T. 2003, PASP, 115, 389

Valenti, J. A., \& Fischer, D. A. 2005, ApJS, 159, 141

Wright, J. T., Fakhouri, O., Marcy, G. W., et al. 2011b, PASP, 123, 412

Wright, J. T., Veras, D., Ford, E. B., et al. 2011a, ApJ, 730, 93

Yasui, C., Kobayashi, N., Tokunaga, A. T., Saito, M., \& Tokoku, C. 2009, ApJ, 705,54 